\title{
A Study on Smart Hierarchical Multimedia Service Traffic Distribution Scheme over E2E Mobility Management System
}

\author{
Farkhod Alisherov ${ }^{1}$ and Byungjoo Park ${ }^{2 *}$ \\ ${ }^{1}$ Department of Multimedia Technologies, Tashkent University of Information \\ Technologies \\ ${ }^{2}$ Department of Multimedia Engineering, Hannam University \\ 133 Ojeong-dong, Daeduk-gu, Daejeon, Korea \\ 1Farkhod.alisherov@gmail.com, ${ }^{2 *}$ bjpark@hnu.kr
}

\begin{abstract}
Due to the increase in mobile devices and multimedia service streaming, traffic in a satisfies QoS (Quality of Service), QoE (Quality of Experience) state is becoming a problem as the traffic load increases abruptly over IPv6 wireless networks. Also, the future development of heterogeneous manganese flow mobility issues of the mobile terminal and Decentralized traffic problem appears to have accelerated. Proxy Mobile Internet Protocol version 6 (PMIPv6) is a network-based mobility management protocol, which allows nodes to keep service connectivity while moving around in the IPv6 Internet. PMIPv6 is different from the host-based mobility management protocol Hierarchical Mobile IPv6, which has the same hierarchical mobility management architecture. PMIPv6 outperforms HMIPv6 due to its ability to avoid mobility signaling sent by the mobile host, but the HMIPv6 reduces the intra-domain signaling. This paper introduces a new mobility agent Advanced Mobile Anchor Point, which reduces the intra-domain signaling when a Mobile Node moves inside the Localized Mobile Domain to reduce signaling and handover latency when moving within the LMD.
\end{abstract}

Keywords: Wireless Mobile Networks, Multimedia Traffic Streaming, Service, HPMIPv6, PMIPv6, Mobility Management

\section{Introduction}

Proxy Mobile IPv6 (PMIPv6) [1] was developed as a Network-based Mobility Support (NMS) protocol for the Internet Engineering Task Force (IETF) that does not require a Mobile Node $(\mathrm{MN})$ to be involved in the mobility support signaling to achieve its own mobility service. Hierarchical Mobile IPv6 [2], on the other hand is a Host-based Mobility Support (HMS) protocol that required the MN to participate in the signaling. PMIPv6 and HMIPv6 both have similar hierarchical mobility management architectures in which the MNs are locally managed within a given domain.

In PMIPv6 a MN is managed by proxy mobility agents, a Local Mobility Anchor (LMA), several Mobility Access Gateways (MAGs), while in HMIPv6 a MN is managed under a Mobility Anchor Point (MAP) with several Access Routers (ARs). HMIPv6 is used to relieve high mobility signaling when a MN hands off in the subnets; it also requires a host-based mobility stack in the MN. PMIPv6 does not require a host-based mobility stack in the $\mathrm{MN}$, irrespective of the handoff frequency. Figure 1 shows HMIPv6 and PMIPv6 architectures which show that both protocols have similar hierarchical mobility support protocols.

* Corresponding Author 


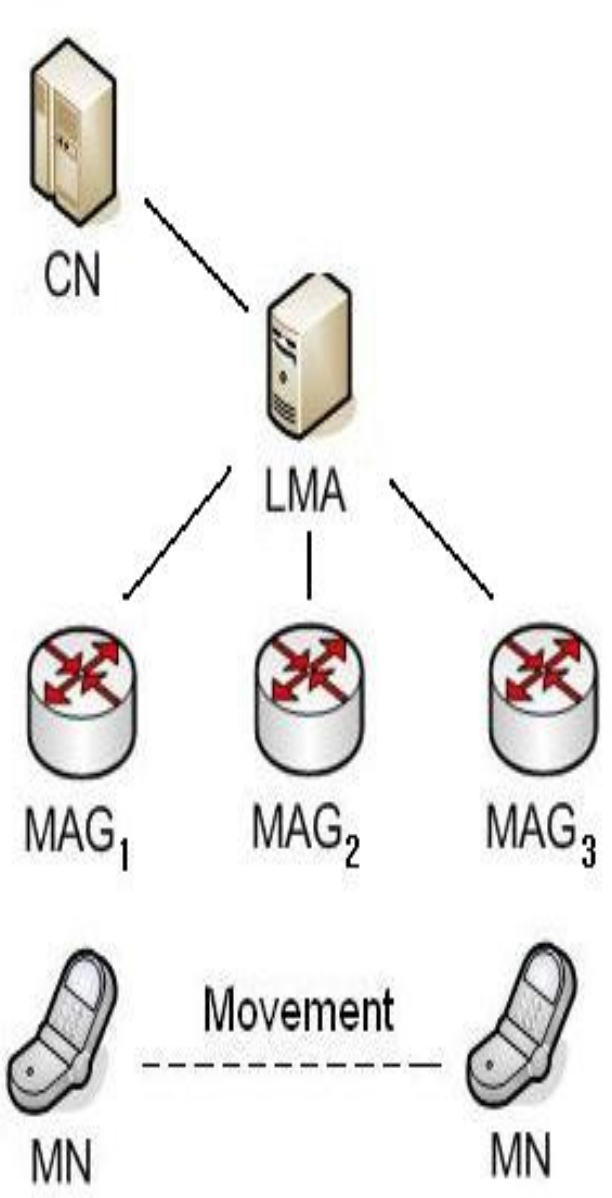

a)
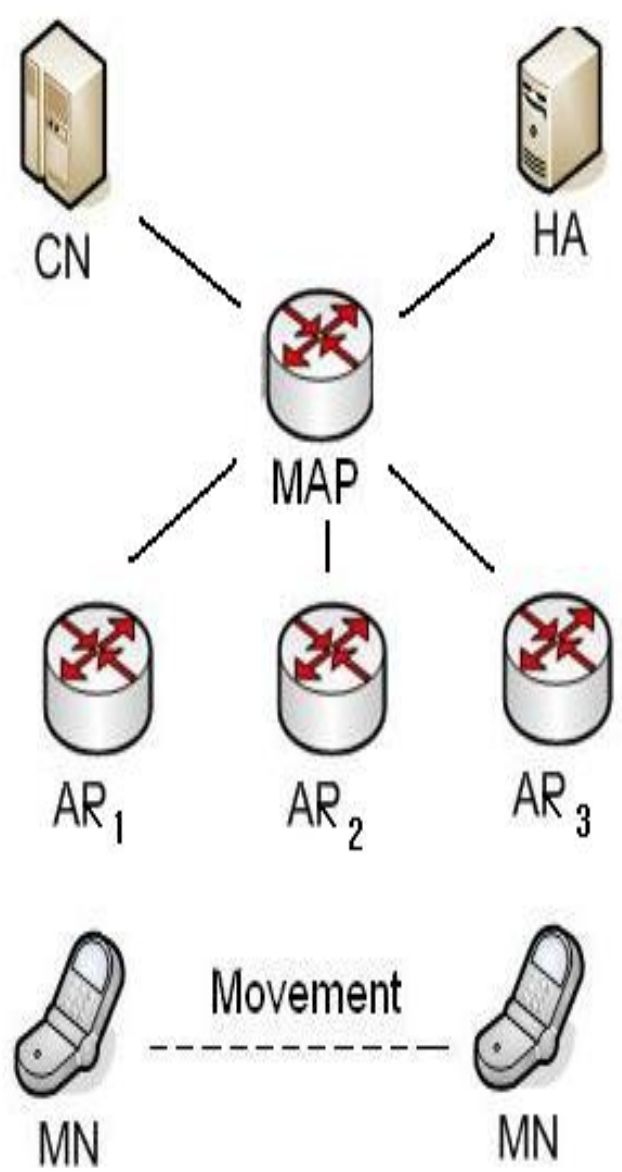

b)

Figure 1. a) PMIPv6 Architecture; b) HMIPv6 Architecture

\section{Related Works and Problem}

\subsection{Mobile IPv6 (MIPv6)}

Mobile IP supports mobility of IP [3] hosts by allowing them to make use of (at least) two IP addresses: a home address that represents the fixed address of the node and a care-of address $(\mathrm{CoA})$ that changes with the IP subnet the mobile node is currently attached to. A correspondent node $(\mathrm{CN})$ that wants to send packets to a mobile node (MN) will send the packets to the MN's home address. In the MN's home network these packets will be intercepted by the home agent and tunneled either directly to the $\mathrm{MN}$ or to a foreign agent to which the MN has a direct link. In MIPv6, each CN can have its own binding cache where home address plus care-of address pairs are stored. This enables route optimization: a $\mathrm{CN}$ is able to send packets directly to a $\mathrm{MN}$ when the $\mathrm{CN}$ has a recent entry for the $\mathrm{MN}$ in its corresponding binding cache.

When a $\mathrm{CN}$ sends a packet directly to a $\mathrm{MN}$, it does not encapsulate the packet as the HA does when receiving a packet from the $\mathrm{CN}$ to be forwarded, but makes use of the IPv6 Routing Header Option. When the $\mathrm{CN}$ does not have a binding cache entry for the MN, it sends the packet to the MN's home address. The MN's home agent will then forward the packet. The MN, when receiving an encapsulated packet, will inform the corresponding $\mathrm{CN}$ about the current $\mathrm{CoA}$. In order to keep the home address to CoA mappings up-to-date, a mobile node has to signal corresponding 
changes to its home agent and/or correspondent nodes when performing a handoff to another IP subnet.

Since in MIPv6 both, HA and CN, maintain binding caches, a common message format called binding updates is used to inform $\mathrm{HA}$ and $\mathrm{CNs}$ about changes in the point of attachment. Additionally, since the BUs have associated a certain lifetime, even if the MN does not change its location a BU to its HA and CNs is necessary before the lifetime expires to keep alive the entry in the binding caches. Binding updates (BU) can be acknowledged by BU Acks (BAck). Mobile IPv6 signaling is done in extension headers that can also be piggybacked on regular packets. To acquire a CoA in Mobile IPv6, a mobile node can build on IPv6 stateless and stateful auto-configuration methods. With The stateless autoconfiguration it is not necessary to contact any entity to obtain a new CoA, reducing the handoff process duration [4].

\subsection{Hierarchical Mobile IPv6 (MIPv6)}

MNs moving quickly as well as far away from their respective home domain or correspondent nodes produce significant BU signaling traffic and will suffer from handoff latency and packet losses when no extension to the baseline Mobile IP protocol is used. Hierarchical Mobile IPv6 (HMIPv6) [2] is a localized mobility management protocol that aims to reduce the signaling load due to user mobility. The mobility management inside the local domain is handled by a Mobility Anchor Point (MAP). Mobility between separate MAP domains is handled by MIPv6.

The MAP basically acts as a local Home Agent. When a mobile node enters into a new MAP domain it registers with it obtaining a regional care-of address ( $\mathrm{RCoA}$ ) and an on-link care-of-address. The RCoA is the address that the mobile node will use to inform its Home Agent and correspondent nodes about its current location. Then, the packets will be sent to and intercepted by the MAP, acting as a proxy, and routed inside the domain to the (LCoA). When a mobile node then performs a handoff between two access points within the same MAP domain only the MAP has to be informed. Note, however that this does not imply any change to the periodic BUs a MN has to sent to HA, CNs and now additionally to the MAP. HMIPv6 presents the following advantages: it includes a mechanism to reduce the signaling load in case of handoffs within the same domain and may improve handoff performance reducing handoff latency and packet losses since intra-domain handoffs are performed locally. However, since the periodic BUs are not reduced but the ones due to handoffs, the gain depends on the mobility of the mobile nodes [5]. 
MN: Mobile Node

PAP: Previous Access Point

PAR: Previous Access Router
NAP: New Access Point

NAR: New Access Router

MAP: Mobility Anchor Point
HA: Home Agent

CN: Correspondent Node

TRA: Triggerd RA

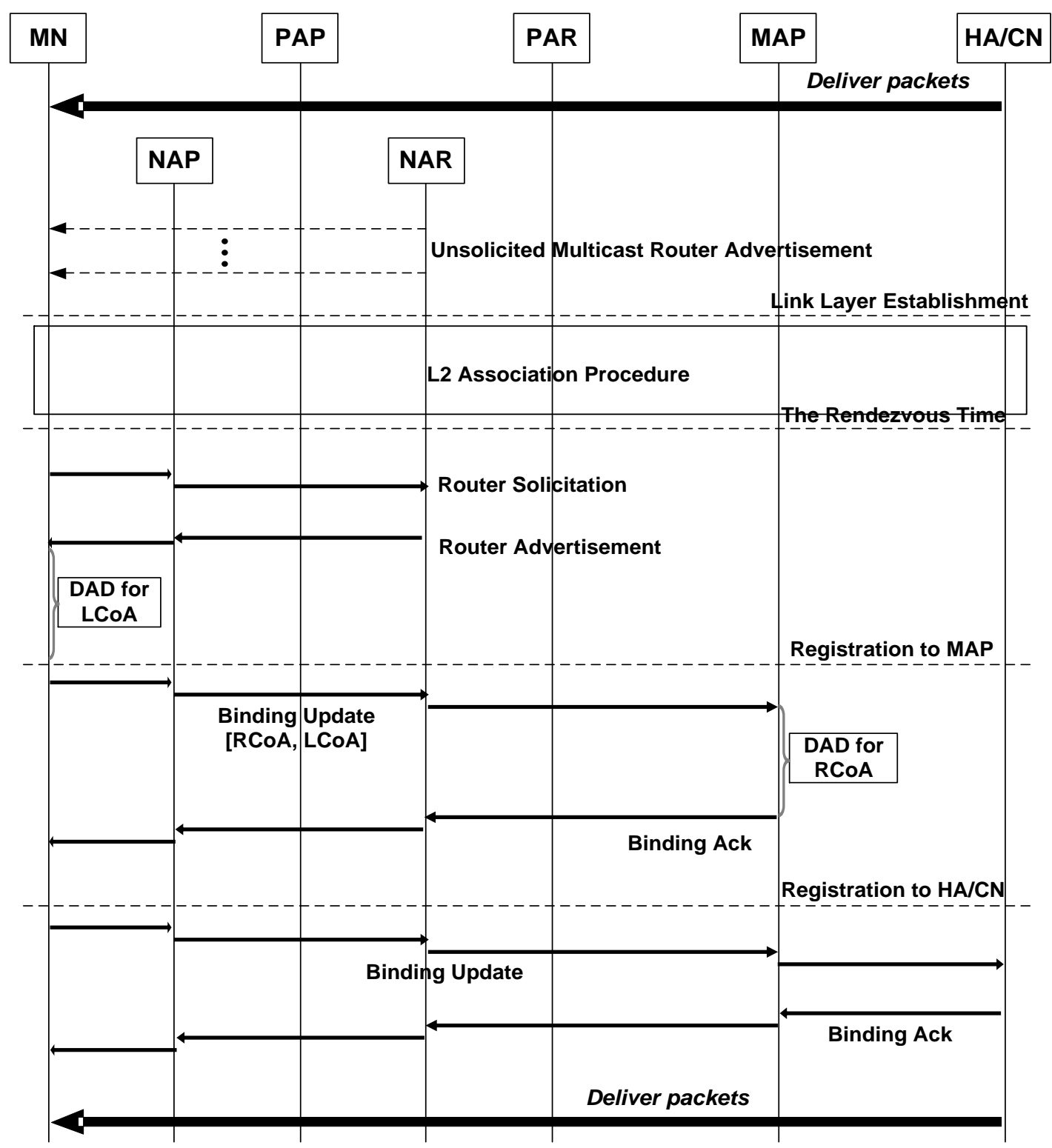

Figure 3. HMIPv6 Handover Signal Flow

\subsection{Proxy Mobile IPv6 (MIPv6)}

PMIPv6 [1] introduces two entities LMA and MAG, which manage all mobility related signaling so $\mathrm{MN}$ is not involved in any mobility related signaling. MN needs to generate only one global address, so as the MN hands off and changes its point of attachment from one MAG to another, the MN still uses the same address which was obtained in the first MAG. Figure 3 shows the signaling flow diagram of a handover in a PMIPv6 domain. 


\section{MN: Mobile Node}

AAA: Policy Store

PMAG: Previous Mobile Access Gateway

NMAG: New Mobile Access Gateway

LMA: Localized Mobility Anchor

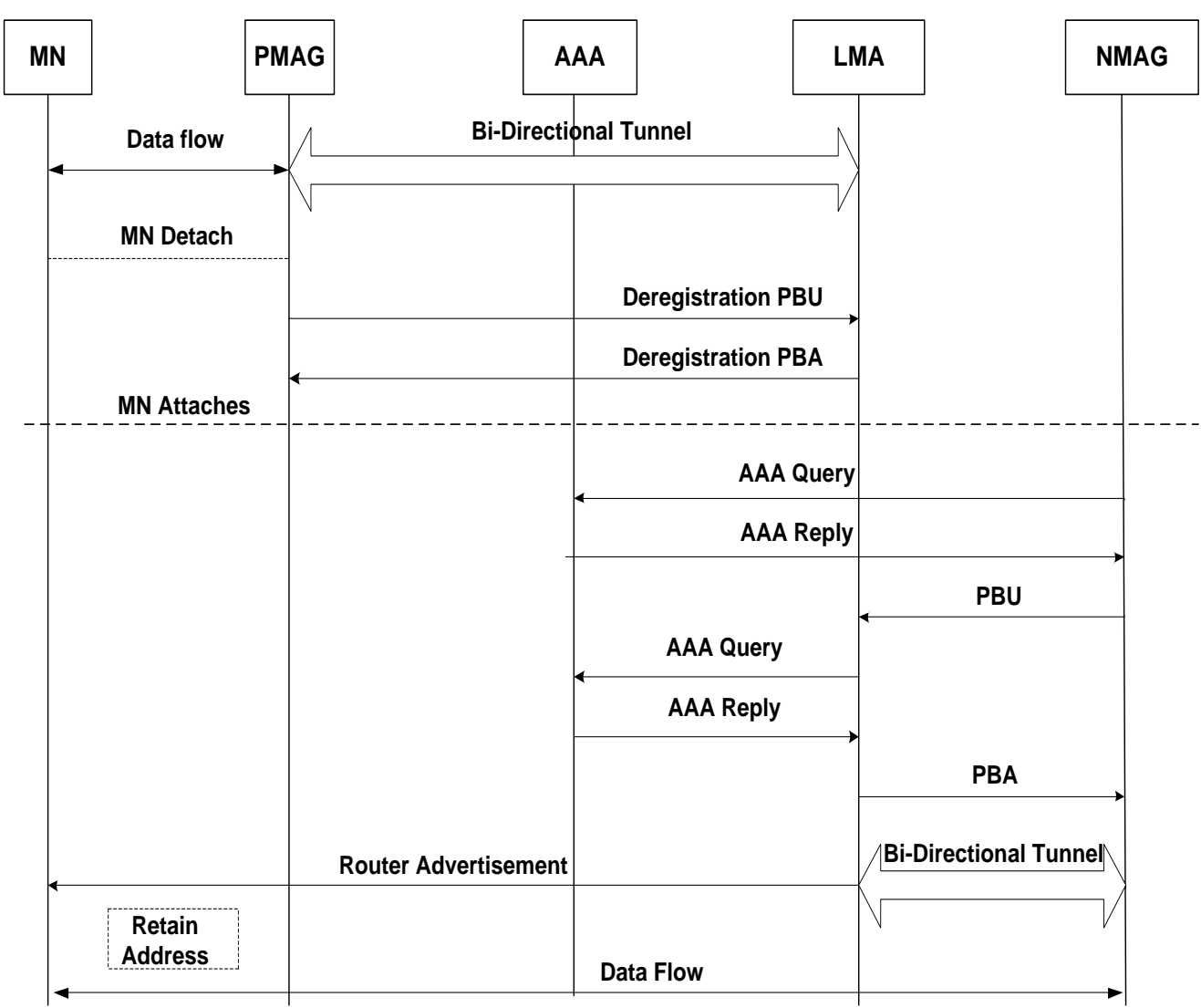

Figure 3. PMIPv6 Handover Signal Flow

The binding registration messages are initiated from the MAG, which is in the network infrastructure, as opposed to HMIPv6 where the same signaling is initiated from the MN. Once the MN enters the PMIPv6 domain, the network ensures that the $\mathrm{MN}$ is always on its home network and can obtain its home address on any access network. Thus, the serving network assigns a unique home network prefix to each $\mathrm{MN}$, and this prefix stays with the MN as long as it moves within the PMIPv6 domain. Consequently, there is no need to re-configure the care-of-address (CoA) at the $\mathrm{MN}$, thus optimizing the handover performance by reducing the handover latency. Furthermore, PMIPv6 reduces the binding update delay by reducing the round-trip-time, thus effectively reducing handover latency. Also, PMIPv6 eliminates the delay introduced by Duplicate Address Detection (DAD) in host based mobility management protocols, hence effectively reducing handover delay [6]. 


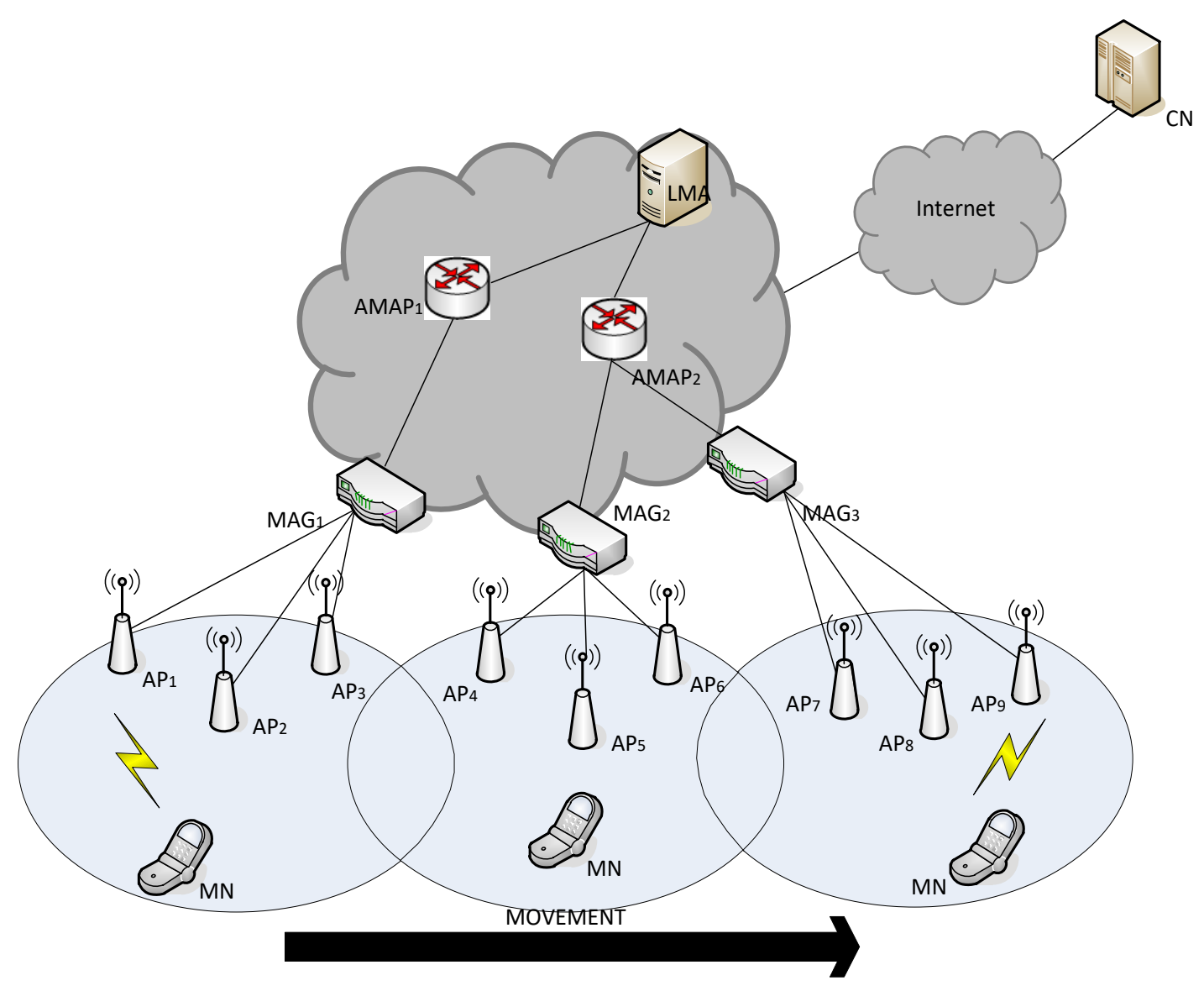

Figure 4. Illustration of the Proposed HPMIPv6

Compress Conditional Access System which provides channel and contents, VOD System, sending video signal, Data Broad System to embody many kinds of two way services, and the signal from a base band with H.264 and make data signals. After multiplex, scrambling, making them an IP and then transfer them.

Backbone Network is network that passes from processed data to process. Namely, there are Multicasting, QoS technology for delivery of contents ensured reliability and efficiency between Head-End and set-top box. Home is transmitted to set-top box of user and possible to use service of IPTV through network-equipment of DS-LAM (Digital Subscriber Line Access Multiplexer) of Access Network.

\section{Smart Hierarchical Multimedia Service Traffic Distribution Scheme over E2E Mobility Management System}

Since the intra-domain and inner-domain handover latencies in Proxy Mobile IPv6 is same, an HMIPv6 MAP entity, AMAP, usage is proposed. As in HMIPv6, AMAP will act as a regional Home address within a Localized Mobility Domain (LMD). And will decrease signaling when the MN hands off within the AMAP between MAGs. 


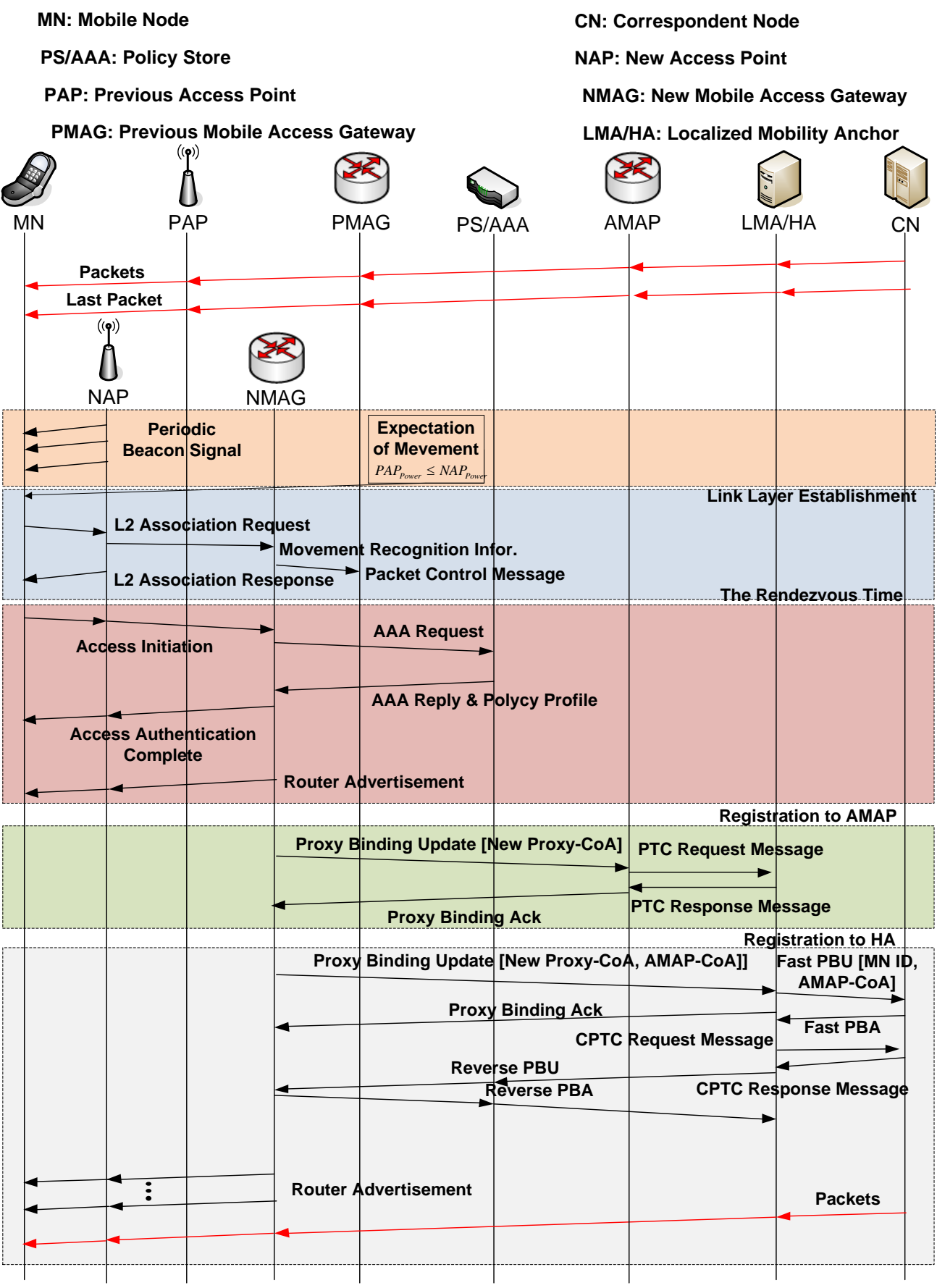

Figure 5. Proposed HPMIPv6 Handover Procedure

Additionally, a second CoA (AMAP-CoA) is required that will be used during hand offs within the AMAP, between MAGs. Figure 4 illustrates a HPMIPv6 domain.

HPMIPv6 handover signaling flow is a follow: (Figure 5)

0) $\mathrm{MN}$ receiving the periodic beacon message that include neighbors MAG's address from NAP. By using L2 beacon signal, MN can compare power signal strength between previous PAP_power and NAP_power. MN send own's information with power result to NMAG by using L2 association request messages. By using this procedure, network can support traffic control. 
1) The MN send L2 Association Request message to NAP. NAP reply L2 Association Response message to establish link layer.

2) The MN send Access Initiation message to NMAG. The NMAG send AAA Request message to PS/AAA server to verify MN's information. PS/AAA send reply message with policy profile to NMAG. The NMAG send Access Authentication Complete message to $\mathrm{MN}$

3) The NMAG detects the attachment of the MN and then sends Proxy Binding Update (PBU) with the new CoA to the AMAP to register the attachment. The AMAP responds with the Proxy Binding Acknowledgement (BU) message as a receipt that the new binding cache has been created.

4) The AMAP send Packet Traffic Control(PTC) Request message to LMA/HA to manage network traffic load which can support seamless packet transmission without packet disruption.

5) The LMA/HA send PTC Response message to AMAP.

6) Similarly, the NMAG also sends a PBU to LMA including the AMAP-CoA address with the Proxy-CoA, so that a bi-directional tunnel can be created between the LMA and AMAP.

7) Upon receiving the PBU the LMA sends a fast PBU with the MN Id and the AMAP-CoA to the Correspondent Node $(\mathrm{CN})$, upon receival, the $\mathrm{CN}$ responds with the Fast PBA. The the LMA sends a PBA to the NMAG as a receipt of a successful binding registry.

8) As soon as receiving FPBA message from $\mathrm{CN}$, the LMA/HA send Current Packet Traffic Control (CPTC) Request message to $\mathrm{CN}$ which can control correspondent transmission packet amount. After alignment packets, the CN send CPTC Response message to LMA/HA.

9) LMA sends a Reverse PBU to the NMAG and receives the Reverse PBA from the NMAG. NMAG creates a transient binding cache entry for the MN. A transient binding cache entry is identical to a normal PMIPv6 binding cache entry except that it could be deleted if the LMA does not receive a normal PBU from the NMAG within a predefined time. That is, a transient binding cache entry has a lifetime associated and its deletion does not need any signaling. The use of Fast PBU/PBA and Reverse PBU/PBA during an MN's handover enables a great control on the fast update of the MN's binding cache entry at LMA and the fast forwarding to the NMAG for the traffic destined for the MN.

10) A bi-direction tunnel is set up between the AMAP and LMA, and then the data packets will be forwarded between the $\mathrm{CN}$ and $\mathrm{MN}$.

11) When the MN moves again, to a new MAG within the AMAP domain, the new MAG needs only to sends a PBU to the AMAP, steps 2-9 are omitted.

\section{Performance Analysis}

It is assumed that during the L2 handover with exchanging periodic beacon signal, we can neglect beacon procedure and time caused by movement recognition information sending procedure. Also, during the regular proxy binding update 
procedures, the PTC procedure, FPBU and CPTC procedures would be processed. That is, the PTC, CPTC and FPBU processing time during total handover latency can be neglected.

The handover latency in PMIPv6 is due to procedures that take place during the handover:

Attachment notification $T_{\text {attach }}=2 t_{1}$, where $\mathrm{t}_{1}$ is the communication time between MN and NAP; There is also a signal weighting factor $a$, since the communication is in the Link Layer. $\left(\alpha \mathrm{T}_{\text {attach }}\right)$;

Authentication Query and Authentication Reply due to the MAG verifying if the $\mathrm{MN}$ is eligible for the network-based mobility management service $T_{\text {auth }}=2 t_{1}+2 t_{2}$ $+2 t_{3}$, where $t_{2}$ is the communication time between NAP and NMAG, and $t_{3}$ is the communication time between NMAG and AAA;

Router Advertisement message delay $T_{r a}=t_{1}+t_{2}$;

Proxy binding registration delay $T_{\text {binding }(L M A)}=2 t_{4}+2 t_{5}$, where $t_{4}$ is the NMAG and LMA communication time and $t_{5}$ is between LMA and AAA;

Follow by another Router Advertisement message sent by the NMAG;

Followed by a Binding registration to the $\mathrm{CN} T_{\text {binding }(C N)}=2 t_{4}+2 t_{6}$, where $\mathrm{t}_{6}$ is the communication time between LMA and $\mathrm{CN}$;

So, the handover latency in Proxy Mobile IPv6: $T_{P M I P v 6}=\alpha T_{\text {attach }}+T_{\text {auth }}+2 T_{\text {ra }}$ $+T_{\text {binding }(L M A)}+T_{\text {binding }(C N)}=\alpha\left(2 t_{1}\right)+4 t_{1}+4 t_{2}+2 t_{3}+4 t_{4}+2 t_{5}+2 t_{6}$. Futhermore, the handover after the movement inside the same LMD domain remains the same, so the total handover latency for the PMIPv6:

$$
T_{P M I P V 6}=2 \alpha\left(2 \mathrm{t}_{1}\right)+8 t_{1}+8 t_{2}+4 t_{3}+8 t_{4}+4 t_{5}+4 t_{6}
$$

The handover latency in the proposed HPMIPv6:

Attachment notification $T_{\text {attach }}=2 t_{1}$, The signal weighting factor $\alpha$, Authetication query and reply $T_{\text {auth }}=2 t_{1}+2 t_{2}+2 t_{3}$, Router Advertisement $T_{r a}=t_{1}+t_{2}$, and the packet retransmission rate $\beta$ are similar as in PMIPv6;

Followed by proxy binding registration to the LMA $T_{\text {binding }(L M A) 2}=2 t_{4}$

Fast PBU $\mathrm{T}_{\text {fast }}=2 \mathrm{t}_{6}$;

Reverse Binding registration to NMAG and AAA $T_{\text {binding(reverse })}=2 t_{4}+2 t_{5}$

So, the handover latency in Hierarchical Proxy Mobile IPv6: $T_{H P M I P v 6}=\alpha T_{\text {attach }}+$ $T_{\text {auth }}+2 T_{\text {ra }}+T_{\text {binding(LMA)2 }}+T_{\text {binding(LMA })}+T_{\text {binding(reverse })}+T_{\text {fast }}=\alpha\left(2 \mathrm{t}_{1}\right)+4 t_{1}+4 t_{2}+$ $4 t_{3}+2 t_{4}+2 t_{5}+2 t_{6}+2 t_{7}$. After the movement inside the LMD, the $\mathrm{T}_{\text {binding(reverse) }}$ and $\mathrm{T}_{\text {binding(LMA)2 }}$ and $\mathrm{T}_{\text {fast }}$ are omitted, so the total latency handover for HPMIPv6:

$T_{H P M I P V 6}=2 \alpha\left(2 \mathrm{t}_{1}\right)+7 t_{1}+7 t_{2}+6 t_{3}+2 t_{4}+2 t_{5}+2 t_{6}$

Proposed HPMIPv6 and PMIPv6 handover latency schemes are compared. It is supposed that every $\mathrm{MN}$ is positioned in the same network and message transmission time is the same in both methods. Table 1 shows Performance Analysis Parameters. 
Table 1. Performance Analysis Parameters

\begin{tabular}{|c|c|c|}
\hline Symbols & Description & Value \\
\hline$t_{1}$ & $\mathrm{MN} \Leftrightarrow \mathrm{NAP}$ & $10 \mathrm{~ms}$ \\
\hline$t_{2}$ & NAP $\Leftrightarrow$ NMAG & $20 \mathrm{~ms}$ \\
\hline$t_{3}$ & $\mathrm{NMAG} \Leftrightarrow \mathrm{AAA}$ & $30 \mathrm{~ms}$ \\
\hline $\mathrm{t}_{4}$ & NMAG $\Leftrightarrow$ LMA & $50 \mathrm{~ms}$ \\
\hline$t_{5}$ & $\mathrm{LMA} \Leftrightarrow \mathrm{AAA}$ & $20 \mathrm{~ms}$ \\
\hline$t_{6}$ & $\mathrm{LMA} \Leftrightarrow \mathrm{CN}$ & $10 \mathrm{~ms}$ \\
\hline a & Signal Weighting factor & $10 \mathrm{~ms}$ \\
\hline
\end{tabular}

\section{Conclusion}

Proposed HPMIPv6 and PMIPv6 handover latency schemes are compared. It is supposed that every $\mathrm{MN}$ is positioned in the same network and message transmission time is the same in both methods. Table 1 shows Performance Analysis Parameters. This paper introduced a new mobility agent Advanced Mobile Anchor Point (AMAP), which reduces the intra-domain signaling when a Mobile Node (MN) moves inside the Localized Mobile Domain (LMD) to reduce signaling and handover latency when moving within the LMD. The performance analysis and comparison show that HPMIPv6 has reduced handover delay because of reduced signaling during inner-domain hand offs between MAGs than the PMIPv6 protocol.

\section{Acknowledgments}

This research was supported by Basic Science Research Program through the National Research Foundation of Korea (NRF) funded by the Ministry of Science, ICT and future planning (2015R1A2A2A03002851).

\section{References}

[1] S. Gundavelli, K. Leung, V. Devarapalli, K. Chowdhury and B. Patil, "Proxy Mobile IPv6. RFC 5213”, (2008) August.

[2] H. Soliman, C. Castelluccia, K. ElMalki and L. Bellier, "Hierarchical Mobile IPv6 (HMIPv6) mobility management", RFC 5380, (2008) October.

[3] D. Johnson and C. Perkins, "Mobility support in IPv6", RFC3775, IETF, (2004) June.

[4] R. Koodili, G. Dommety, A. Yegin, C. Perkins, G. Tsirtsis, K. El-Malki, M. Khalil, "Fast Handoffs for Mobile Ipv6", RFC4068. IETF, (2005) July.

[5] H. Young Jung, "Fast Handover for Hierarchical MIPv6 (FHMIPv6)", IETF draft-jungmobopts-fhmipv6-00.txt, (2005).

[6] H. Yokota, K. Chowdhury and R. Koodli, "Fast Handovers for Proxy Mobile IPv6", draftietf-mipshop-pfmipv6-08.txt IETF Internet draft, (2009). 